\title{
On the Mean Dimensions of Restricted Random Walks
}

\author{
Peter H. Verdier and Edmund A. DiMarzio
}

\author{
Institute for Materials Research, National Bureau of Standards, Washington, D.C. 20234
}

(October 25, 1968)

\begin{abstract}
A simple method is presented for obtaining mean dimensions of certain restricted random walks on lattices.

Key Words: Lattice; nonreversing walk; polymer dimension; random walk.
\end{abstract}

Random walks in three dimensions are frequently used as models of the possible configurations of certain forms of long-chain polymer molecules in solution [1]. ${ }^{1}$ In particular, walks are often used in which restrictions are imposed upon the angle between successive steps in the walk. Such restricted random walks are, of course, Markovian, and standard methods [2] are available for obtaining their mean dimensions. It is the purpose of this note to point out, however, that for a certain class of restricted random walks on a lattice, mean dimensions may be obtained by a simpler method which involves little more than an appropriate counting of configurations. The walks in question consist of steps of fixed length, with an equal-odds choice made at each step from a set of possible steps consisting of: a set of steps possessing a rotation-inversion symmetry axis, decreased or augmented by a step whose direction is determined by the direction of the preceding step. As a simple example, we consider the nonreversing walk on a simple cubic lattice of side unity in three dimensions. Here the choice at each step is made, with equal probability, from among five steps, four of them perpendicular to the last step, and the fifth continuing in the same direction. By way of example, we shall calculate the mean-square end-to-end length of this walk in two ways.

We start by writing

$$
\left\langle l^{2}\right\rangle=\left\langle\left(\mathbf{r}_{1}+\ldots+\mathbf{r}_{N}\right)^{2}\right\rangle=N+2 \sum_{j=1}^{N-1} \sum_{i=1}^{N-j}\left\langle\mathbf{r}_{i} \cdot \mathbf{r}_{i+j}\right\rangle,
$$

where $\left\langle l^{2}\right\rangle$ is the mean-square end-to-end length of the walk, $N$ is the number of steps, and the vector $\mathbf{r}_{i}$ represents the $i$ th step. Let us consider the average dot product $\left\langle\mathbf{r}_{i} \cdot \mathbf{r}_{i+j}\right\rangle$. For the lattice in question, since there are five choices at each step, there are $5^{j}$ configurations over which to average. We can sort these out in two different ways, suggested by the identities:

$$
\begin{aligned}
5^{j} & =(4+1)^{j}=4^{j}+j \cdot 4^{j-1}+\ldots+1=\sum_{k=0}^{j}\left(\begin{array}{l}
j \\
k
\end{array}\right) 4^{j-k} \\
& =(6-1)^{j}=6^{j}-j \cdot 6^{j-1}+\ldots+(-1)^{j} \\
& =\sum_{k=0}^{j}\left(\begin{array}{l}
j \\
k
\end{array}\right) 6^{j-k}(-1)^{k} .
\end{aligned}
$$

In the first case, we regard the choices for each step as consisting of the set of four steps perpendicular to the last step, plus a step in the same direction. Then it is clear that in the first of the binomial expansions above, the first term is the number of $j$-step walks consisting entirely of rightangle steps, the second term is the number of walks with $j-1$ right-angle steps and one parallel step, and so forth. If we perform the sum of $\mathbf{r}_{i} \cdot \mathbf{r}_{i+j}$ over the $5^{j}$ configurations by summing over each

\footnotetext{
${ }^{1}$ Figures in brackets indicate the literature references at the end of this paper.
} 
of these sets, we find that the sum over each set except the last is zero. To see this, we note that to every walk with a right-angle step in it there corresponds another walk produced by inverting the portion of the original walk that follows the right angle. Since $\mathbf{r}_{i+j}$ points in opposite directions for the two walks, their total contribution to $\left\langle\mathbf{r}_{i} \cdot \mathbf{r}_{i+j}\right\rangle$ is zero. (A similar argument may be made for lattices with a higher-order rotation-inversion axis, such as the triangular lattice in two dimensions.) Thus, the sum over the $5^{j}$ configurations reduces to the single term in the last set, which corresponds to the configuration with all steps parallel. For this configuration, $\mathbf{r}_{i} \cdot \mathbf{r}_{i+j}$ is unity, so we have $\left\langle\mathbf{r}_{i} \cdot \mathbf{r}_{i+j}\right\rangle=(1 / 5)^{j}$.

The same conclusion is also reached by regarding the choices at each set as consisting of the isotropic set of six choices, less the choice which constitutes reversal of the previous step. In this case, the first term in the second binomial expansion corresponds to completely unrestricted sixchoice walks, the second term to walks with at least one reversal produced by forcing a reversal at one step of an otherwise unrestricted walk, and so on. As before, the contributions from all sets except the last are zero, and we are again left with the contribution from the single member of the last set, taken with coefficient $(-1)^{j}$. However, since this term corresponds to the configuration with a forced reversal at every step, the dot product will itself be $(-1)^{j}$, and we shall again have the result $\left\langle\mathbf{r}_{i} \cdot \mathbf{r}_{i+j}\right\rangle=(1 / 5)^{j}$. It is interesting to note that the sole contribution to the sum of the dot product over all configurations which do not reverse may be expressed in terms of the dot product value for the configuration which violates the restriction at every step.

Once $\left\langle\mathbf{r}_{i} \cdot \mathbf{r}_{i+j}\right\rangle$ has been obtained, evaluation of $\left\langle l^{2}\right\rangle$ is straightforward. Since by definition of the random process $\left\langle\mathbf{r}_{i} \cdot \mathbf{r}_{i+j}\right\rangle$ is dependent only on the number $j$ of intervening steps; we rewrite eq (1) in the form:

$$
\left\langle l^{2}\right\rangle=N+2 \sum_{j=1}^{N-1}(N-j)\left\langle\mathbf{r}_{i} \cdot \mathbf{r}_{i+j}\right\rangle=N+2 \sum_{j=1}^{N-1}(N-j)(1 / 5)^{j} .
$$

Now, for $x \neq 1$, we have

$$
\sum_{j=1}^{N-1}(N-j) x^{j}=N x /(1-x)-x\left(1-x^{N}\right) /(1-x)^{2}
$$

so that we can write

$$
\left\langle l^{2}\right\rangle=N[1+2 x /(1-x)]-2 x\left(1-x^{N}\right) /(1-x)^{2},
$$

or with $x=1 / 5,\left\langle l^{2}\right\rangle=(3 / 2) N-(5 / 8)\left[1-(1 / 5)^{N}\right]$, in agreement with the result obtained for this walk by Domb and Fisher [3].

The foregoing argument may be applied to any isotropic nonreversing walk, in any number of dimensions. The result will be given by eq (3), with $x$ equalling the reciprocal of the number of choices at each step. The argument can be applied equally well to what might be called a noncontinuing random walk, where continuation in the direction of the preceding step is barred, but the other possibilities, including reversal, are allowed. For such a walk, eq (3) again applies, but $x$ is now the negative of the reciprocal of the number of allowed choices.

The same technique may be applied to walks where the choice is made from an isotropic set plus or minus two (or in principle, more) steps which depend upon the last step. As a trivial example, consider a random walk on a simple square or cubic lattice with both continuation and reversal disallowed. In this case, the last term in the appropriate binomial expansion is $(-2)^{j}$, and of this even number of terms, half have $\mathbf{r}_{i+j}$ pointing in the same direction as $\mathbf{r}_{i}$ and half have it pointing in the opposite direction. Hence all the nondiagonal dot products vanish, and we have immediately the result $\left\langle l^{2}\right\rangle=N$. In the two-dimensional case, this result can, of course, be verified directly when $N$ is even by redrawing the steps in pairs to form an unrestricted walk of $N / 2$ steps, each of length $\sqrt{2}$.

\section{References}

[1] See, for example, Flory, P. J., Principles of Polymer Chemistry (Cornell University Press, Ithaca, N.Y., 1953).

[2] Flory, P. J., op. cit., ch. X.

[3] Domb, C., and Fisher, M. E., Proc. Camb. Phil. Soc. 54, 48 (1958). 